\title{
Analysis of Critical Velocities for an Infinite Timoshenko Beam Resting on an Elastic Foundation Subjected to a Harmonic Moving Load
}

\author{
Bin Zhen, ${ }^{1}$ Wei Luo, ${ }^{2}$ and Jian $\mathrm{Xu}^{3}$ \\ ${ }^{1}$ School of Environment and Architecture, University of Shanghai for Science and Technology, Shanghai 200093, China \\ ${ }^{2}$ College of Hydraulic and Environmental Engineering, China Three Gorges University, Yichang 443002, China \\ ${ }^{3}$ School of Aerospace Engineering and Mechanics, Tongji University, Shanghai 200092, China \\ Correspondence should be addressed to Wei Luo; ctgulunwen@163.com
}

Received 28 February 2014; Accepted 27 June 2014; Published 14 July 2014

Academic Editor: Toshiaki Natsuki

Copyright (C) 2014 Bin Zhen et al. This is an open access article distributed under the Creative Commons Attribution License, which permits unrestricted use, distribution, and reproduction in any medium, provided the original work is properly cited.

\begin{abstract}
Critical velocities are investigated for an infinite Timoshenko beam resting on a Winkler-type elastic foundation subjected to a harmonic moving load. The determination of critical velocities ultimately comes down to discrimination of the existence of multiple real roots of an algebraic equation with real coefficients of the 4th degree, which can be solved by employing Descartes sign method and complete discrimination system for polynomials. Numerical calculations for the European high-speed rail show that there are at most four critical velocities for an infinite Timoshenko beam, which is very different from those gained by others. Furthermore, the shear wave velocity must be the critical velocity, but the longitudinal wave velocity is not possible under certain conditions. Further numerical simulations indicate that all critical velocities are limited to be less than the longitudinal wave velocity no matter how large the foundation stiffness is or how high the loading frequency is. Additionally, our study suggests that the maximum value of one group velocity of waves in Timoshenko beam should be one "dangerous" velocity for the moving load in launching process, which has never been referred to in previous work.
\end{abstract}

\section{Introduction}

Moving-load problems have received a great deal of attention worldwide in the past several decades. The earliest moving-load problems are about railway bridges excited by traveling trains. Then the application areas gradually have been extended to high-speed commuter trains, missile sled test tracks, high-speed projectile launchers, and so on. "Critical velocity" is a phenomenon that has been found in beams subjected to moving loads. A resonant wave in a beam can be induced when a load moves at the critical velocity, which results in an unbounded increase of the displacements, rotation, and bending moments of the beam for an undamped case. In reality, damping always exists, but even so, a very large deflection of the beam may occur if the moving load approaches the critical velocity [1-3]. Researches show that the "critical velocity" phenomenon may shorten life of launchers due to high stress, fatigue, premature wear, and gouging of rails [4]. Therefore, it is very necessary to determine the critical velocity for a beam subjected to a moving load.

Timoshenko [3] firstly derived an expression for the critical velocity of a concentrated load moving along the Bernoulli-Euler beam resting on a continuous elastic foundation. It was proposed that the critical velocity is analogous to an additional longitudinal compressive force causing beam buckling. By analyzing a Timoshenko beam resting on an elastic foundation and subjected to a concentrated load traveling along its length, Crandall [5] indicated that the Timoshenko beam model produced a total of three critical velocities. The study of Florence [6] found that a semiinfinite Timoshenko beam under a moving concentrated load exhibited a singular behavior when the load speed was equal to the shear wave speed or longitudinal wave speed. Steele [7] investigated the same problem but showed that the solution was bounded when the load speed was equal to the shear 
or longitudinal wave speed. Furthermore, his study indicated that the behavior of the Timoshenko beam is quite similar to that of the Bernoulli-Euler beam for load speed somewhat less than the shear wave speed. Chonan [8] showed that the frequency of the moving load has considerable effect on the dynamical behavior of the Timoshenko beam. Then, later researchers usually investigated critical velocity problem by considering a harmonic load moving on the Timoshenko beam. Chen et al. [9] discussed the critical velocity of an infinite Timoshenko beam to a harmonic moving load by establishing its dynamic stiffness matrix. The dynamic response of a European high-speed railway subjected to a harmonic moving load was calculated to show that there is only a critical velocity when the frequency of the harmonic load $\omega$ is equal to zero. Moreover, two critical velocities approached one as the loading frequency approached zero, and they separated from the critical velocity for $\omega=0$ more and more as $\omega$ increases; one is decreasing and the other is increasing from the critical velocity for $\omega=0$, respectively. Further numerical simulations showed that the critical velocity increases as the foundation stiffness increases for a given loading frequency.

According to previous statements, the knowledge about critical velocities for an infinite Timoshenko beam under a moving load is still not completely clear. First, no consensus has been reached yet even for the number of the critical velocities for the beam subjected to a harmonic moving load. Second, it is still worth to be discussed whether or not the shear and longitudinal wave velocities are the critical velocities. Third, the velocity of moving load traveling on a Timoshenko beam in previous study was just limited to less than $1.1 \mathrm{~km} / \mathrm{s}$ [9]. However, one concern in the launching process is gouging, a form of damage that occurs in the range of $1.5-1.8 \mathrm{~km} / \mathrm{s}$, which limits the rail life [10]. Thus, the distribution of critical velocities faster than $1.1 \mathrm{~km} / \mathrm{s}$ needs to be analyzed for optimization of the railgun barrel design.

To address the three problems mentioned above, this paper focuses on critical velocities for an infinite Timoshenko beam resting on a Winkler-type elastic foundation subjected to a harmonic moving load. By using the Fourier transform method, the determination of critical velocities for Timoshenko beam ultimately comes down to discrimination of the existence of multiple real roots of an algebraic equation with real coefficients of 4 th degree. The main tools used here are Descartes sign method and complete discrimination system for polynomials [11]. Our research suggests that there may be at most four critical velocities if the lowest phase velocity of waves in the Timoshenko beam is less than the shear wave velocity. Moreover, the shear wave velocity must be one critical velocity, but the longitudinal wave velocity is not possible for the Timoshenko beam under certain conditions. Additionally, numerical simulations for the European highspeed rail indicate that the longitudinal wave velocity is the ultimate velocity limit of the critical velocity for the Timoshenko beam regardless of how large the foundation stiffness is or how high the frequency of the harmonic moving load is.

The rest of the paper is organized as follows. In Section 2, complete discrimination system for polynomials is introduced briefly. In Section 3, the critical velocities for an infinite
Timoshenko beam resting on an elastic foundation under a harmonic moving load are investigated. Numerical simulations are carried out to verify the correctness of analysis results in Section 4. Conclusions are drawn in Section 5.

\section{Complete Discrimination System for Polynomials}

Given a polynomial $f(x)$ with real coefficients,

$$
f(x)=m_{0} x^{n}+m_{1} x^{n-1}+\cdots+m_{n}
$$

its derivative is written as

$$
f^{\prime}(x)=m_{0} n x^{n-1}+m_{1}(n-1) x^{n-2}+\cdots+m_{n-1} .
$$

$\operatorname{Discr}(f)$ denotes the Sylvester matrix of $f(x)$ and $f^{\prime}(x)$ [11, 12] and $D_{k}(f) ; k=1,2, \ldots, n$, denotes the determinant of the submatrix of $\operatorname{Discr}(f)$ formed by the first $2 k$ rows and the first $2 k$ columns. If the number of the sign changes of the discriminant sequence

$$
\left\{D_{1}(f), D_{2}(f), \ldots, D_{n}(f)\right\}
$$

is $v$, then the number of the pairs of distinct conjugate imaginary roots of $f(x)$ equals $v$. Furthermore, if the number of nonvanishing members of the list is $l$, then the number of the distinct real roots of $f(x)$ equals $l-2 v$.

\section{Analysis of Critical Velocities for an Infinite Timoshenko Beam under a Harmonic Moving Load}

Consider an infinite Timoshenko beam resting on a Winklertype elastic foundation subjected to a harmonic moving load. Its governing equation for an undamped case can be expressed by [13]:

$$
\begin{gathered}
E I \frac{\partial^{2} \psi}{\partial x^{2}}+\mu G A\left(\frac{\partial y}{\partial x}-\psi\right)=\rho I \frac{\partial^{2} \psi}{\partial t^{2}} \\
\mu G A\left(\frac{\partial^{2} y}{\partial x^{2}}-\frac{\partial \psi}{\partial x}\right)+p \delta(x-v t) e^{i \omega t}-k y=m \frac{\partial^{2} y}{\partial t^{2}}
\end{gathered}
$$

in which $y=y(x, t)$ and $\psi=\psi(x, t)$ are beam deflection and slope. $E, G, A, I, k, \mu, \rho$, and $m$ are the modulus of elasticity, shear modulus, cross-sectional area of the beam, cross-sectional moment of inertia, the stiffness of the foundation per unit length, sectional shear coefficient, beam material density, and the mass of the beam per unit length, respectively. $p e^{i \omega t} \delta(x-v t)$ represents a harmonically varying load with the frequency $\omega$ moving at a constant velocity $v$, $i=\sqrt{-1}$, and $\delta(\cdot)$ is the Dirac's delta function. 
Eliminating $y$, (4) becomes (the analysis result is the same if $\psi$ is eliminated in (4))

$$
\begin{aligned}
\frac{\partial^{4} \psi}{\partial t^{4}} & +\frac{E \mu G A}{m \rho} \frac{\partial^{4} \psi}{\partial x^{4}}-\frac{k E}{m \rho} \frac{\partial^{2} \psi}{\partial x^{2}}+\left(\frac{k}{m}+\frac{\mu G A}{\rho I}\right) \frac{\partial^{2} \psi}{\partial t^{2}} \\
& -\left(\frac{\mu G A}{m}+\frac{E}{\rho}\right) \frac{\partial^{4} \psi}{\partial x^{2} \partial t^{2}}+\frac{k \mu G A}{m \rho I} \psi \\
= & \frac{\mu G A}{m \rho I} \frac{\partial\left[p \delta(x-v t) e^{i \omega t}\right]}{\partial x} .
\end{aligned}
$$

Applying the following positive and inverse Fourier transforms to (5)

$$
\begin{gathered}
\widehat{\psi}(\eta, t)=\int_{-\infty}^{+\infty} \psi(x, t) e^{-i \eta x} d x, \\
\psi(x, t)=\frac{1}{2 \pi} \int_{-\infty}^{+\infty} \widehat{\psi}(\eta, t) e^{i \eta x} d \eta,
\end{gathered}
$$

where $\eta$ is the transformed field of $x$, then the stable-state response (slope) of the Timoshenko beam can be expressed by

$$
\psi(x, t)=-\frac{1}{2 \pi} \int_{-\infty}^{+\infty} \frac{p \mu G A}{m \rho I \Phi(\eta)} e^{i \eta(x-v t) e^{i \omega t}} d \eta,
$$

where

$\Phi(\eta)=b_{1} \eta^{4}+b_{2} \eta^{3}+b_{3} \eta^{2}+b_{4} \eta+b_{5}$,

$b_{1}=\left(v^{2}-v_{c 1}^{2}\right)\left(v^{2}-v_{c 2}^{2}\right), \quad b_{2}=2 \omega v\left(v_{c 1}^{2}+v_{c 2}^{2}-2 v^{2}\right)$,

$b_{3}=\left(6 \omega^{2}-\omega_{c 1}^{2}-\omega_{c 2}^{2}\right) v^{2}+\omega_{c 1}^{2} v_{c 2}^{2}-\left(v_{c 1}^{2}+v_{c 2}^{2}\right) \omega^{2}$,

$b_{4}=2 \omega v\left(\omega_{c 1}^{2}+\omega_{c 2}^{2}-2 \omega^{2}\right), \quad b_{5}=\left(\omega^{2}-\omega_{c 1}^{2}\right)\left(\omega^{2}-\omega_{c 2}^{2}\right)$.

$v_{c 1}=\sqrt{\mu G A / m}, v_{c 2}=\sqrt{E / \rho}$ are the shear and longitudinal wave velocities, respectively; $\omega_{c 1}=\sqrt{k / m}, \omega_{c 2}=\sqrt{\mu \mathrm{GA} / \rho I}$ are two "cutoff" frequencies [14] in the Timoshenko beam. The descriptions about the phase and group velocities of waves in the Timoshenko beam are given in Appendix below.

Our goal is to determine critical velocities of the moving load at which the steady-state beam displacement $\psi$ is infinite. Therefore, the load velocity related to the divergence of integral (7) is the critical velocity. The integrand in (7) decreases as $\eta \rightarrow \pm \infty$, so the divergence of the integral cannot be related to integration at infinity. Thus, the only possibility for the divergence of integrand in (7) is that $\Phi(\eta)$ has real zeros. Furthermore, the real zeros have to be of the second or higher order, since for the simple real zero the integral converges in the Cauchy principle sense $[15,16]$. A detailed discussion for $\Phi(\eta)$ possessing real zeros of at least the second order is as follows.

(1) Consider $\omega=0$.

Equation (8) can be written as

$$
\Phi(\eta)=b_{1} \eta^{4}+d_{1} \eta^{2}+d_{2}
$$

where $b_{1}$ is defined by $(8), d_{1}=\omega_{c 1}^{2} v_{c 2}^{2}-\left(\omega_{c 1}^{2}+\omega_{c 2}^{2}\right) v^{2}$, and $d_{2}=\omega_{c 1}^{2} \omega_{c 2}^{2}$.

If $v=v_{c 1}$ or $v_{c 2}$, (9) can only have simple real zeros. Thus, the shear and longitudinal wave velocities $v_{c 1}, v_{c 2}$ cannot be critical velocities when $\omega=0$.

If $v \neq v_{c 1}, v_{c 2}$, based on complete discrimination system for polynomials, the discriminant sequence of (9) is given by

$$
\left[1,-b_{1} d_{1}, E_{1}, E_{2}\right] \text {, }
$$

where $E_{1}=b_{1} d_{1}\left(4 b_{1} d_{2}-d_{1}^{2}\right)$ and $E_{2}=b_{1} d_{2}\left(4 b_{1} d_{2}-d_{1}^{2}\right)^{2}$. Then, the multiplicities of real zeros of (9) are greater than or equal to 2 if and only if $E_{1}=E_{2}=0$ and $b_{1} d_{1}<0$. Solving the equation $E_{1}=E_{2}=0$, one has

$$
v_{c r}=\sqrt{\frac{\omega_{c 1}\left(-a_{1} \omega_{c 1} \pm 2 v_{c 1} \omega_{c 2}^{2} \sqrt{a_{2}}\right)}{\left(\omega_{c 2}^{2}-\omega_{c 1}^{2}\right)^{2}}},
$$

where $a_{1}=2 \omega_{c 2}^{2} v_{c 1}^{2}+\left(\omega_{c 2}^{2}-\omega_{c 1}^{2}\right) v_{c 2}^{2}$ and $a_{2}=\omega_{c 1}^{2} v_{c 1}^{2}+\left(\omega_{c 2}^{2}-\right.$ $\left.\omega_{c 1}^{2}\right) v_{c 2}^{2}$. The critical velocity defined by (11) also has to satisfy the condition

$$
\left(v_{\mathrm{cr}}^{2}-v_{c 1}^{2}\right)\left(v_{\mathrm{cr}}^{2}-v_{c 2}^{2}\right)\left(v_{\mathrm{cr}}^{2}-\frac{\omega_{c 1}^{2} v_{c 2}^{2}}{\omega_{c 1}^{2}+\omega_{c 2}^{2}}\right)>0 .
$$

(2) Consider $\omega>0$ and $v=v_{c 1}$.

Equation (8) can be expressed by

$$
\Phi(\eta)=e_{1} \eta^{3}+e_{2} \eta^{2}+e_{3} \eta+b_{5},
$$

where $e_{1}=2 \omega v_{c 1}\left(v_{c 2}^{2}-v_{c 1}^{2}\right), e_{2}=\left(5 v_{c 1}^{2}-v_{c 2}^{2}\right) \omega^{2}-\left(\omega_{c 1}^{2}+\omega_{c 2}^{2}\right) v_{c 1}^{2}+$ $\omega_{c 1}^{2} v_{c 2}^{2}, e_{3}=2 \omega v_{c 1}\left(\omega_{c 1}^{2}+\omega_{c 2}^{2}-2 \omega^{2}\right)$, and $b_{5}$ is defined by $(8)$.

The discriminant sequence of (13) is given by

$$
\left[1, e_{2}^{2}-3 e_{1} e_{3}, E_{5}\right],
$$

where $E_{5}=-27 b_{5}^{2} e_{1}^{2}+2\left(9 e_{2} b_{5}-2 e_{3}^{2}\right) e_{3} e_{1}+e_{2}^{2}\left(e_{3}^{2}-4 b_{5}\right)$. Equation (13) has multiple real zeros if and only if $E_{5}=0$. Considering the relation between the modulus of elasticity $E$ and shear modulus $G: G=E / 2(1+\nu)$, where $v$ is Poisson's ratio, one has $v_{c 1}=\sqrt{\mu G A / m}=\sqrt{\mu / 2(1+\nu)} \sqrt{E / \rho}=\alpha v_{c 2}$. In addition, letting $\omega_{c 1}=\beta \omega_{c 2}, E_{5}$ can be rewritten as

$$
E_{5}(\omega)=4 v_{c 2}^{6}\left(r_{1} \omega^{10}+r_{2} \omega^{8}+r_{3} \omega^{6}+r_{4} \omega^{4}+r_{5} \omega^{2}+r_{6}\right),
$$

where $r_{1}=\left(\alpha^{2}-1\right)^{2}, r_{2}=\omega_{c 2}^{2}\left[\left(4 \beta^{2}+3\right) \alpha^{4}+14 \alpha^{2}-1-4 \beta^{2}\right]$, $r_{3}=\omega_{c 2}^{4}\left[\alpha^{6} \beta^{2}+\left(3-24 \beta^{2}+6 \beta^{4}\right) \alpha^{4}+\left(-11-29 \beta^{2}+4 \beta^{4}\right) \alpha^{2}+\right.$ $\left.2 \beta^{2}\left(2+3 \beta^{2}\right)\right], r_{4}=\omega_{c 2}^{6}\left[3 \beta^{2}\left(1+\beta^{2}\right) \alpha^{6}+\left(12 \beta^{2}+1+4 \beta^{6}-\right.\right.$ $\left.\left.24 \beta^{4}\right) \alpha^{4}+\left(25+19 \beta^{2}\right) \beta^{2} \alpha^{2}-2 \beta^{4}\left(2 \beta^{2}+3\right)\right], r_{5}=\omega_{c 2}^{8} \beta^{2}\left[\left(3 \beta^{4}-\right.\right.$ $\left.21 \beta^{2}+3\right) \alpha^{6}+\left(\beta^{6}+39 \beta^{2}-14\right) \alpha^{4}-\left(7 \beta^{2}+2 \beta^{4}+17\right) \beta^{2} \alpha^{2}+$ $\left.\beta^{4}\left(4+\beta^{2}\right)\right]$, and $r_{6}=\omega_{c 2}^{10} \beta^{2}\left(\alpha^{2} \beta^{2}+\alpha^{2}-\beta^{2}\right)^{3}$.

The shear wave velocity $v_{c 1}$ is the critical velocity as long as $E_{5}(\omega)=0$ has positive real roots. It is easy to verify that $E_{5}\left(0.8 \omega_{c 2}\right) E_{5}\left(\omega_{c 2}\right)<0$ for $0<\alpha<1 / 2$ and $0<\beta<$ $\sqrt{2} / 2$, which means that equation $E_{5}(\omega)=0$ has at least one positive real root in $\left[0.8 \omega_{c 2}, \omega_{c 2}\right]$. Thus, the shear wave velocity $v_{c 1}$ must be the critical velocity for the Timoshenko beam provided that $0<\alpha<1 / 2$ and $0<\beta<\sqrt{2} / 2$. 

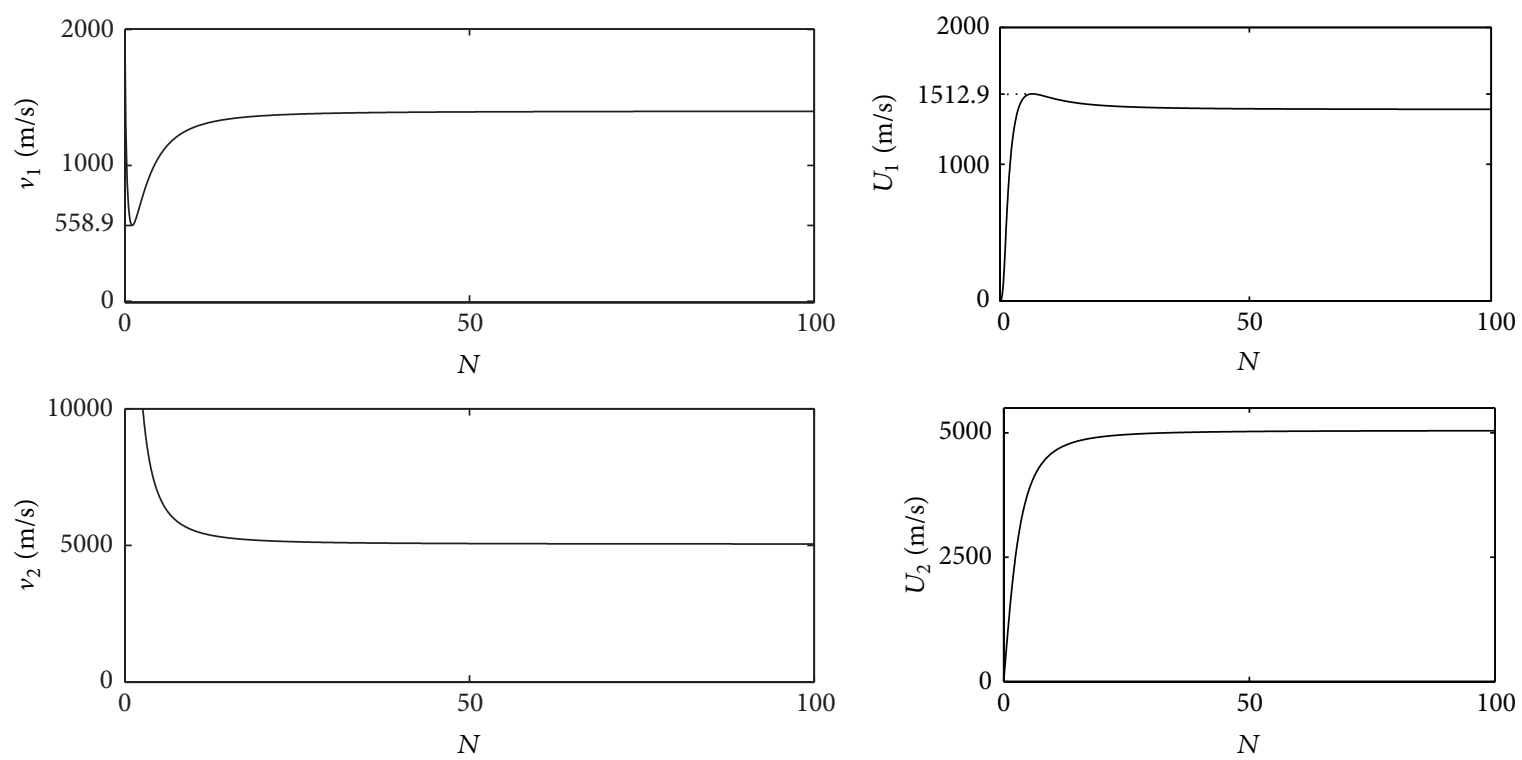

(a) The curves of the phase velocities $v_{1}, v_{2}$ varying with wave number $\mathrm{N}$, and the lowest phase velocity of naturally propagating waves in the Timoshenko beam is $v_{1 \mathrm{~min}}=558.9 \mathrm{~m} / \mathrm{s}$

(b) The curves of the group velocities $U_{1}, U_{2}$ varying with wave number $N$, and the maximum value of the group velocity $U_{1}$ is $U_{1 \max }=1512.9 \mathrm{~m} / \mathrm{s}$

FIGURE 1: The change curves of the phase and group velocities of naturally propagating waves versus wave number $N$ in the Timoshenko beam for $k=1.6 \times 10^{7} \mathrm{~N} / \mathrm{m}^{2}$.

(3) Consider $\omega>0$ and $v=v_{c 2}$.

Equation (8) can be given by

$$
\Phi(\eta)=e_{4} \eta^{3}+e_{5} \eta^{2}+e_{6} \eta+b_{5}
$$

where $e_{4}=2 \omega v_{c 2}\left(v_{c 1}^{2}-v_{c 2}^{2}\right), e_{5}=\left(5 v_{c 2}^{2}-v_{c 1}^{2}\right) \omega^{2}-\omega_{c 2}^{2} v_{c 2}^{2}$, $e_{6}=2 \omega v_{c 2}\left(\omega_{c 1}^{2}+\omega_{c 2}^{2}-2 \omega^{2}\right)$, and $b_{5}$ is defined by (8).

The discriminant sequence of (16) is derived by

$$
\left[1, e_{5}^{2}-3 e_{4} e_{6}, E_{6}\right]
$$

where $E_{6}=-27 b_{5}^{2} e_{4}^{2}+2\left(9 e_{5} b_{5}-2 e_{6}^{2}\right) e_{6} e_{4}+e_{5}^{2}\left(e_{6}^{2}-4 b_{5}\right)$. Equation (16) has multiple real zeros if and only if $E_{6}=0$. By letting $\alpha=v_{c 1} / v_{c 2}$ and $\beta=\omega_{c 1} / \omega_{c 2}, E_{6}$ can be written as

$$
E_{6}=4 v_{c 2}^{6}\left(r_{7} \omega^{10}+r_{8} \omega^{8}+r_{9} \omega^{6}+r_{10} \omega^{4}+r_{11} \omega^{2}+r_{12}\right),
$$

where $r_{7}=\alpha^{2}\left(\alpha^{2}-1\right)^{2}, r_{8}=\omega_{c 2}^{2}\left[\left(11 \alpha^{4}-\alpha^{6}-11 \alpha^{2}+1\right) \beta^{2}+\right.$ $\left.\left(3-\alpha^{4}+14 \alpha^{2}\right) \alpha^{2}\right], r_{9}=\omega_{c 2}^{4}\left[8\left(4 \alpha^{2}-\alpha^{4}-1\right) \beta^{4}+\left(-52 \alpha^{4}+\right.\right.$ $\left.\left.4+\alpha^{6}-\alpha^{2}\right) \beta^{2}+\left(3-11 \alpha^{2}\right) \alpha^{2}\right], r_{10}=\omega_{c 2}^{6}\left[3 \beta^{2}\left(12 \beta^{2}+13\right) \alpha^{4}\right.$ $\left.+\left(-16 \beta^{6}-28 \beta^{4}+1-5 \beta^{2}\right) \alpha^{2}+2 \beta^{2}\left(8 \beta^{4}-8 \beta^{2}+3\right)\right], r_{11}=$ $\omega_{c 2}^{8} \beta^{2}\left[\left(-27 \alpha^{4}+36 \alpha^{2}-8\right) \beta^{2}-15 \alpha^{2}+4\right]$, and $r_{12}=\omega_{c 2}^{10} \beta^{2}$.

It is easily checked that $r_{i}>0(i=7,8, \ldots, 12)$ for $0<\alpha<1 / 2$ and $0<\beta<\sqrt{2} / 2$. Based on Descartes sign method, equation $E_{6}=0$ has no positive real root if $r_{i}>0(i=7,8, \ldots, 12)$, which suggests that the longitudinal wave velocity $v_{c 2}$ must not be the critical velocity for the Timoshenko beam if $0<\alpha<1 / 2$ and $0<\beta<\sqrt{2} / 2$.
(4) Consider $\omega>0$ and $v \neq v_{c 1}, v_{c 2}$.

The discriminant sequence of (8) is given by

$$
\left[1, F_{1}, F_{2}, F_{3}\right] \text {, }
$$

where $F_{1}=3 b_{2}^{2}-8 b_{1} b_{3}, F_{2}=\left(16 b_{5} b_{3}-18 b_{4}^{2}\right) b_{1}^{2}+\left(-6 b_{2}^{2} b_{5}+\right.$ $\left.14 b_{3} b_{4} b_{2}-4 b_{3}^{3}\right) b_{1}+b_{2}^{2}\left(b_{3}^{2}-3 b_{2} b_{4}\right)$, and $F_{3}=256 b_{5}^{3} b_{1}^{3}+$ $\left(-192 b_{4} b_{5}^{2} b_{2}+144 b_{3} b_{5} b_{4}^{2}-27 b_{4}^{4}-128 b_{3}^{2} b_{5}^{2}\right) b_{1}^{2}+\left(144 b_{3} b_{5}^{2} b_{2}^{2}-\right.$ $\left.6 b_{2}^{2} b_{5} b_{4}^{2}-80 b_{3}^{2} b_{4} b_{2} b_{5}+18 b_{3} b_{4}^{3} b_{2}+16 b_{3}^{4} b_{5}-4 b_{3}^{3} b_{4}^{2}\right) b_{1}-b_{2}^{2}\left[27 b_{5}^{2} b_{2}^{2}\right.$ $\left.+\left(4 b_{4}^{3}-18 b_{4} b_{5} b_{3}\right) b_{2}-b_{3}^{2} b_{4}^{2}+4 b_{3}^{3} b_{5}\right]$.

Based on complete discriminant system for polynomials, (8) has multiple real zeros if and only if $F_{3}=0$. The critical velocity can be derived by solving $F_{3}=0$ for a given loading frequency $\omega$.

\section{Numerical Simulations}

To verify the validity of our analysis results on critical velocities, the parameters of an European high-speed rail are used to carry out numerical simulations: $E=2 \times 10^{7} \mathrm{~N} / \mathrm{cm}^{2}$, $G=7.69 \times 10^{6} \mathrm{~N} / \mathrm{cm}^{2}, I=3.06 \times 10^{3} \mathrm{~cm}^{4}, m=60.34 \mathrm{~kg} / \mathrm{m}$, $A=76.86 \mathrm{~cm}^{2}, \mu=0.2$, and $\rho=m / A$.

First, consider that the foundation stiffness is taken as $k=1.6 \times 10^{7} \mathrm{~N} / \mathrm{m}^{2}$. The change curves of the phase and group velocities of naturally propagating waves $\left(v_{1,2}, U_{1,2}\right)$ versus wave number $N$ in Timoshenko beam are presented in Figure 1 based on (A.5) in Appendix. As shown, the minimum value of the phase velocity $v_{1}$ is $v_{1 \mathrm{~min}}=558.9$, and the maximum value of the group velocity $U_{1}$ is $U_{1 \max }=1512.9$. In addition, the shear and longitudinal wave velocities in Timoshenko beam are $v_{c 1}=\sqrt{\mu G A / m}=1399.7$ and 


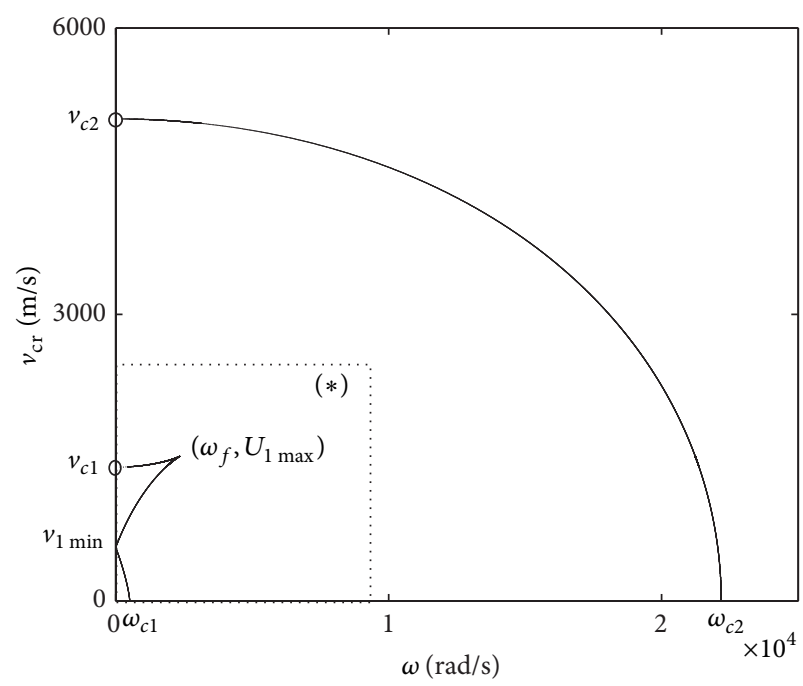

(a)

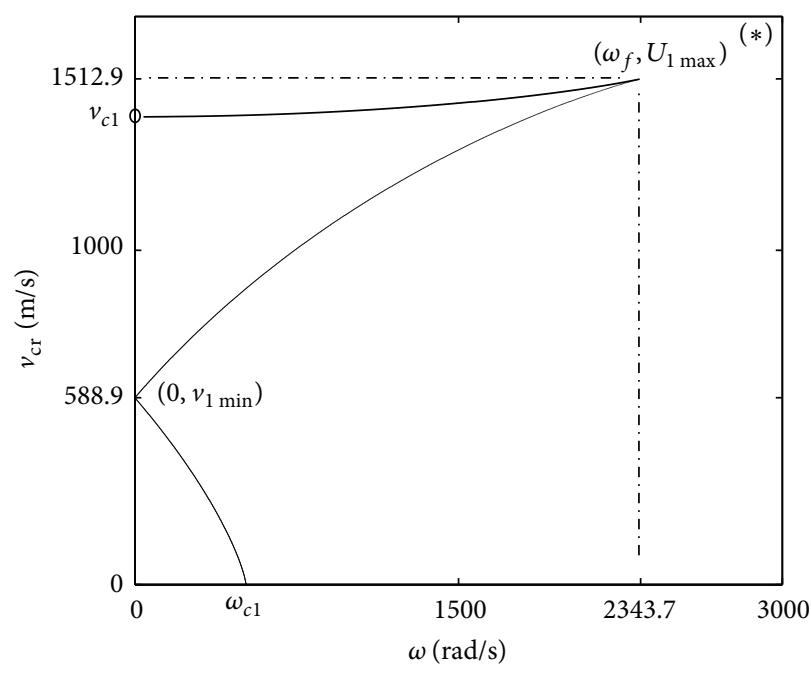

(b)

FIGURE 2: Critical velocity $v_{\text {cr }}$ versus loading frequency $\omega$ for $k=1.6 \times 10^{7} \mathrm{~N} / \mathrm{m}^{2}$.

$v_{c 2}=\sqrt{E / \rho}=5047.3$, and the two cutoff frequencies are $\omega_{c 1}=\sqrt{k / m}=514.9$ and $\omega_{c 2}=\sqrt{\mu G A / \rho I}=22182.7$. Obviously, $\alpha=v_{c 1} / v_{c 2}=0.2773<1 / 2$ and $\beta=\omega_{c 1} / \omega_{c 2}=$ $0.0232<\sqrt{2} / 2$. According to the analysis results in Section 3 , it can be divided into several cases as below.

(1) If loading frequency $\omega=0$, critical velocity $v_{\mathrm{cr}}=$ $v_{1 \text { min }}$. Furthermore, shear and longitudinal wave velocities cannot be the critical velocity for $\omega=0$.

(2) The shear wave velocity $v_{c 1}$ is the critical velocity if loading frequency satisfies $\omega=1835.5$ or 21372.1 .

(3) The longitudinal wave velocity $v_{c 2}$ cannot be the critical velocity for any loading frequency $\omega$.

(4) If $\omega$ and $v \neq v_{c 1}, v_{c 2}$, critical velocity $v_{c r}$ can be determined by solving equation $F_{3}=0$, where $F_{3}$ is defined by (19).

The plot of critical velocity $v_{\text {cr }}$ versus loading frequency $\omega$ in Timoshenko is shown in Figure 2, in which $\omega_{f}$ is the resonant loading frequency obtained by solving equation $F_{3}=0$ (defined by (19)) with $v=U_{1 \max }$.

From Figure 2, there is only one critical velocity at $v_{\mathrm{cr}}=$ $v_{1 \text { min }}$ when loading frequency $\omega=0$; as $\omega$ is increasing, two critical velocities separate from $v_{1 \text { min }}$ more and more; one decreases and vanishes when $\omega>\omega_{c 1}$ and the other increases and vanishes when $\omega>\omega_{f}$. Beyond that, two other critical velocities approach the shear and longitudinal wave velocities $v_{c 1}, v_{c 2}$, respectively, when $\omega$ approaches zero. As $\omega$ is growing, one critical velocity increases from $v_{c 1}$ and disappears when $\omega>\omega_{f}$; the other decreases from $v_{c 2}$ and disappears when $\omega>\omega_{c 2}$. It is shown that there are at most four critical velocities for the Timoshenko beam when $k=$ $1.6 \times 10^{7} \mathrm{~N} / \mathrm{m}^{2}$.

Next, consider that the foundation stiffness $k$ has a wide range changing from $5 \times 10^{6}$ to $1 \times 10^{10} \mathrm{~N} / \mathrm{m}^{2}$. One cutoff frequency $\omega_{c 1}$ will increase with increasing the foundation stiffness $k$, while in the other cutoff frequency $\omega_{c 2}$, the shear and longitudinal wave velocities $v_{c 1}, v_{c 2}$ remain unchanged. The relationships between the critical velocity $v_{\mathrm{cr}}$ and the loading frequency $\omega$ when $k=5 \times 10^{6}, 1 \times 10^{8}, 1 \times 10^{9}$, $1 \times 10^{10} \mathrm{~N} / \mathrm{m}^{2}$, respectively, are shown in Figures 3(a)-6(a). The dependencies of phase and group velocities $\left(v_{1}, U_{1}\right)$ of naturally propagating waves in the Timoshenko beam on wave number $N$ are shown in Figures 3(b)-6(b) accordingly. In Figures 3-6, $v_{1 \text { min }}$ and $U_{1 \text { max }}$ still represent the minimum value of the phase velocity $v_{1}$ and the maximum value of the group velocity $U_{1}$, respectively.

In accordance with Figures 3-6, $v_{1 \text { min }}$ increases and $U_{1 \max }$ decreases with the increase of the foundation stiffness $k$ in the Timoshenko beam. $v_{1 \text { min }}$ is always the critical velocity for loading frequency $\omega=0$ provided that $v_{1 \mathrm{~min}}$ is less than the shear wave velocity $v_{c 1}$. Meanwhile, $U_{1 \max }>v_{c 1}$ when $v_{1 \text { min }}<v_{c 1}$ (Figures 3(b)-5(b)). Once the shear wave velocity $v_{c 1}$ becomes the minimum phase velocity (or $U_{1 \max }<$ $\left.v_{c 1}\right)$, there is no critical velocity for $\omega=0$ and there are at most two critical velocities for a given loading frequency (see Figure 6). From Figures 3(b)-5(b), it should be noted that the critical velocity, which approaches the longitudinal wave velocity $v_{c 2}$ when $\omega=0$ and decreases with increasing $\omega$ and then disappears when $\omega>\omega_{c 2}$, does not change with the foundation stiffness in the range $5 \times 10^{6} \leq k \leq 1 \times 10^{10} \mathrm{~N} / \mathrm{m}^{2}$.

To demonstrate the correctness of our numerical results, the influences of the change of foundation stiffness $k$ on critical velocity $v_{\mathrm{cr}}$ and loading frequency $\omega$ are shown in Figure 7 , in which $k, v_{\mathrm{cr}}$, and $\omega$ are restricted in the region of $5 \times 10^{6} \leq k \leq 1 \times 10^{10} \mathrm{~N} / \mathrm{m}^{2}, v_{\mathrm{cr}}<2 \mathrm{~km} / \mathrm{s}$, and $\omega<$ $4 \times 10^{3} \mathrm{rad} / \mathrm{s}$. Comparing our results which are presented in Figure 7 with that obtained by Chen et al. [9], our calculation results for $v_{\mathrm{cr}}<1.1 \mathrm{~km} / \mathrm{s}$ and $\omega<500 \mathrm{rad} / \mathrm{s}$ are exactly the same as that derived by them. However, their research paper did not give any details about the distribution of critical velocities in the range of $v_{\mathrm{cr}}>1.1 \mathrm{~km} / \mathrm{s}$ and $\omega>500 \mathrm{rad} / \mathrm{s}$. 


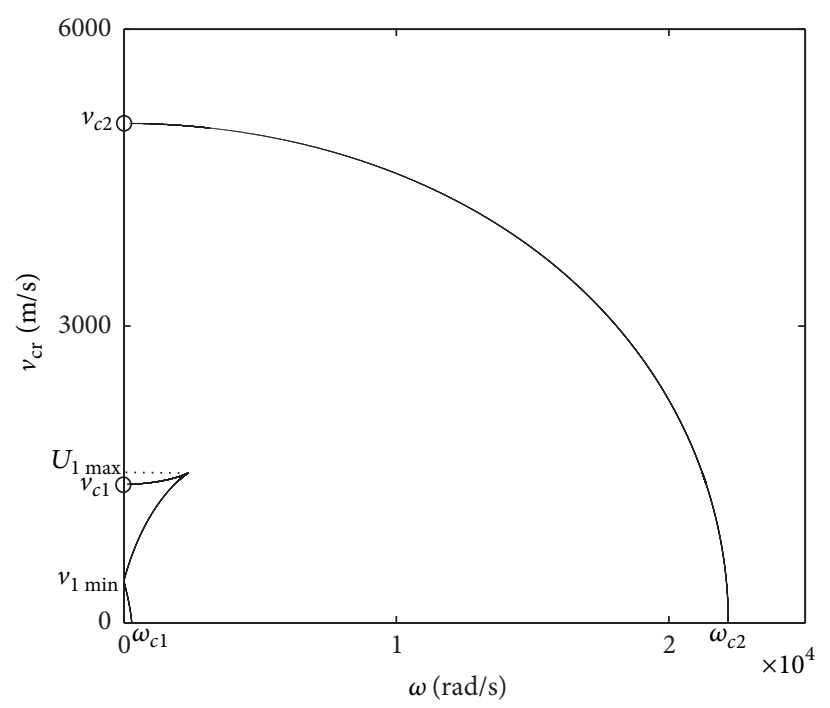

(a) Critical velocity $v_{\text {cr }}$ versus loading frequency $\omega$, in which $v_{1 \text { min }}=$ $422.4 \mathrm{~m} / \mathrm{s}<v_{c 1}, U_{1 \max }=1516.1 \mathrm{~m} / \mathrm{s}>v_{c 1}$, as shown in Figure 3(b)
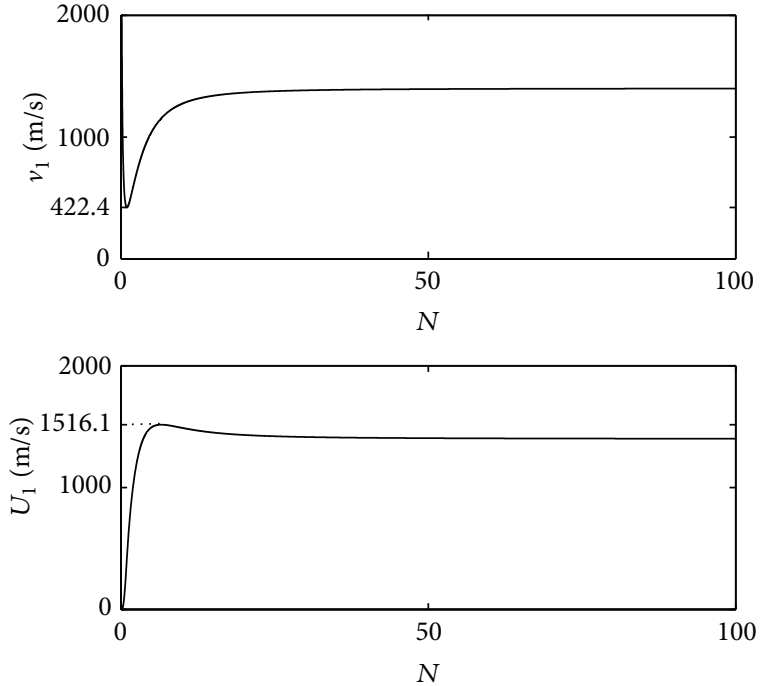

(b) The phase velocity $v_{1}$ and group velocity $U_{1}$ of naturally propagating waves versus wave number $N, v_{1 \min }=422.4 \mathrm{~m} / \mathrm{s}$, $U_{1 \max }=1516.1 \mathrm{~m} / \mathrm{s}$

FIGURE 3: Critical velocity $v_{\text {cr }}$ varies with loading frequency $\omega$ for $k=5 \times 10^{6} \mathrm{~N} / \mathrm{m}^{2}$.

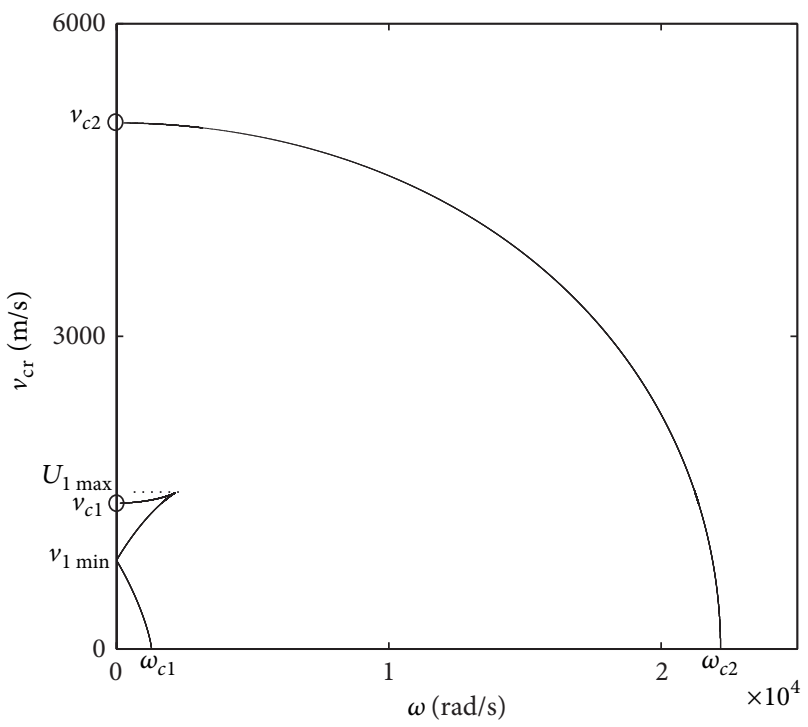

(a) Critical velocity $v_{\text {cr }}$ versus loading frequency $\omega$, in which $v_{1 \text { min }}=$ $851.4 \mathrm{~m} / \mathrm{s}<v_{c 1}, U_{1 \max }=1498.6 \mathrm{~m} / \mathrm{s}>v_{c 1}$, as shown in Figure 4(b)
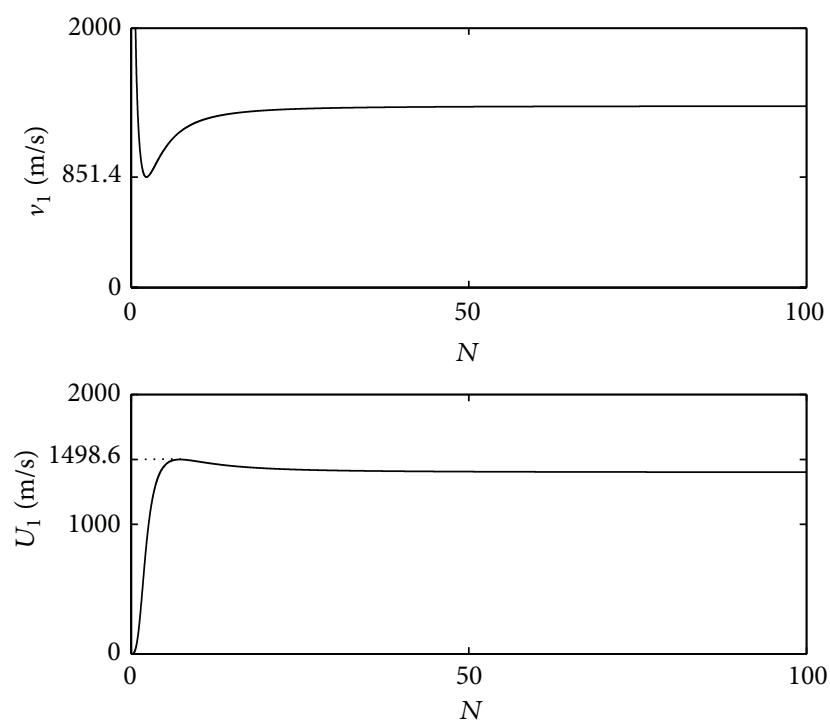

(b) The phase velocity $v_{1}$ and group velocity $U_{1}$ of naturally propagating waves versus wave number $N, v_{1 \min }=851.4 \mathrm{~m} / \mathrm{s}, U_{1 \max }=1498.6 \mathrm{~m} / \mathrm{s}$

FIGURE 4: Critical velocity $v_{\text {cr }}$ varies with loading frequency $\omega$ for $k=1 \times 10^{8} \mathrm{~N} / \mathrm{m}^{2}$.

\section{Conclusions}

Critical velocity phenomenon in the Timoshenko beam has received extensive concern over the past decades. In the past research, there is no unified understanding of the number of critical velocities for the Timoshenko beam. Some scholars considered that the number of critical velocities is three; however, others argued that it should be one or two. Furthermore, there is no general agreement whether or not the shear and longitudinal wave velocities in the Timoshenko beam are the critical velocities. Additionally, an important form of damage in launchers is gouging of rails, which occurs when the velocity of the moving load is in the range of $1.5-1.8 \mathrm{~km} / \mathrm{s}$. Nevertheless, few researches focus on critical velocities faster than $1.1 \mathrm{~km} / \mathrm{s}$ for the Timoshenko beam. To clarify these problems mentioned above, in this paper, critical velocities are analyzed for an infinite Timoshenko beam resting on a Winkler-type elastic foundation subjected to a harmonic moving load. Some conclusions drawn from our study are given as follows. 


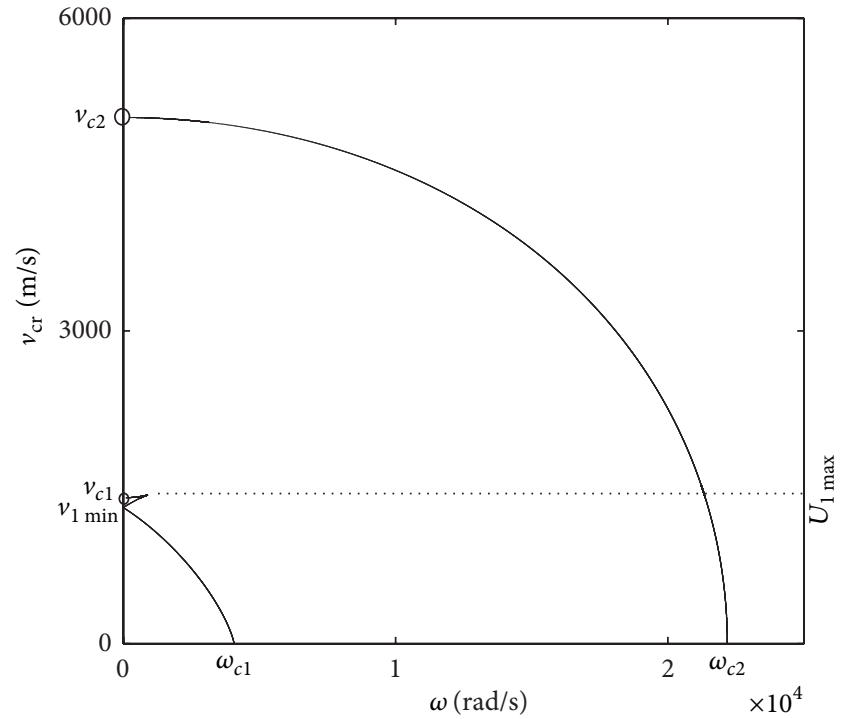

(a) Critical velocity $v_{\text {cr }}$ versus loading frequency $\omega$, in which $v_{1 \mathrm{~min}}$ $1305.4 \mathrm{~m} / \mathrm{s}<v_{c 1}, U_{1 \max }=1425.7 \mathrm{~m} / \mathrm{s}>v_{c 1}$, as shown in Figure $5(\mathrm{~b})$
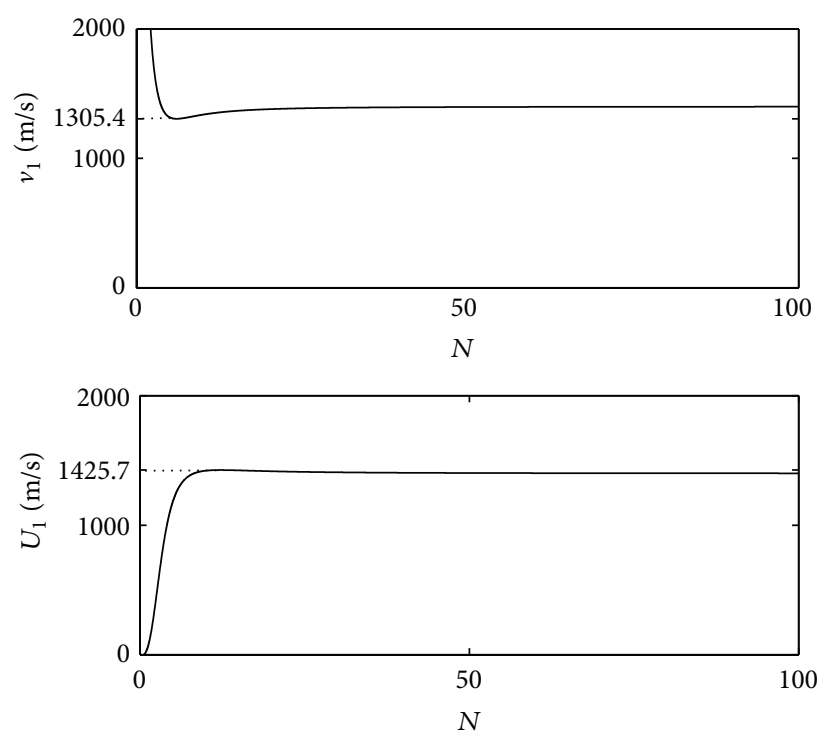

(b) The phase velocity $v_{1}$ and group velocity $U_{1}$ of naturally propagating waves versus wave number $N, v_{1 \min }=1305.4 \mathrm{~m} / \mathrm{s}, U_{1 \max }=1425.7 \mathrm{~m} / \mathrm{s}$

FIGURE 5: Critical velocity $v_{\text {cr }}$ varies with loading frequency $\omega$ for $k=1 \times 10^{9} \mathrm{~N} / \mathrm{m}^{2}$.

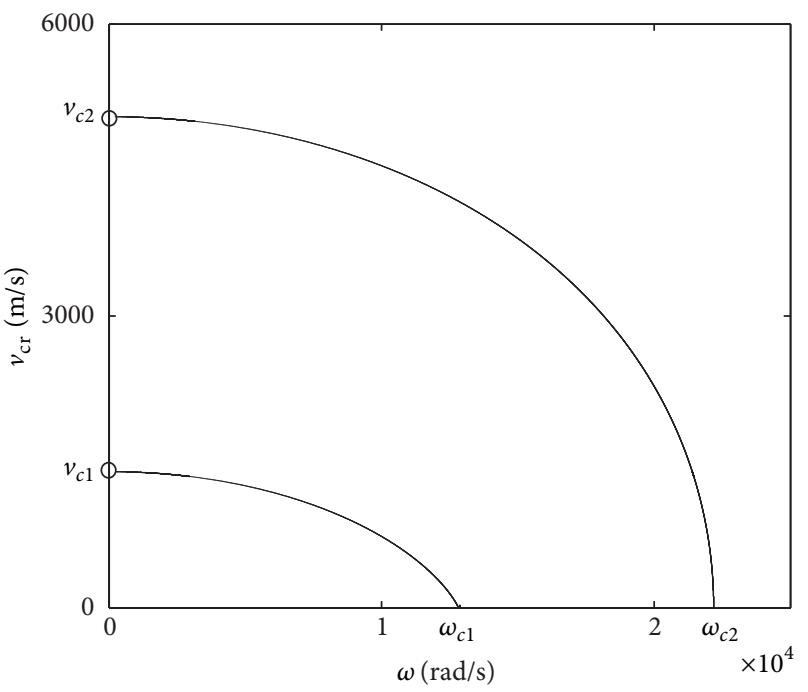

(a) Critical velocity $v_{\text {cr }}$ versus loading frequency $\omega$
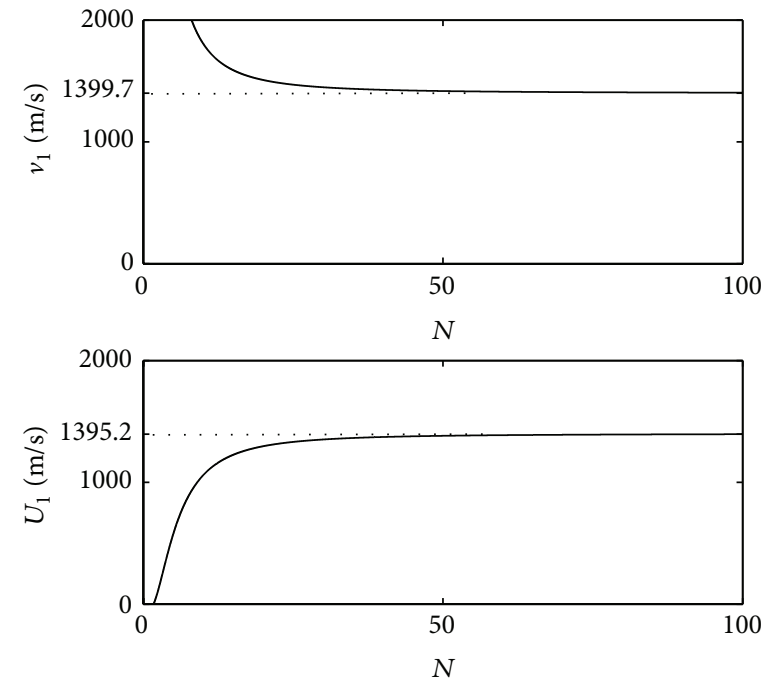

(b) The phase velocity $v_{1}$ and group velocity $U_{1}$ of naturally propagating waves versus wave number $N, v_{1 \min }=v_{c 1}=1399.7 \mathrm{~m} / \mathrm{s}$, $U_{1 \max }=1395.2 \mathrm{~m} / \mathrm{s}<v_{c 1}$

Figure 6: Critical velocity $v_{\text {cr }}$ varies with loading frequency $\omega$ for $k=1 \times 10^{10} \mathrm{~N} / \mathrm{m}^{2}$.

(1) Numerical simulations for the European high-speed rail show that the maximum number of critical velocities for the Timoshenko beam is four if the lowest phase velocity of waves is less than the shear wave velocity, while the maximum number of critical velocities is two if the lowest phase velocity of waves is equal to the shear wave velocity. The number of critical velocities changes with the frequency of the moving load from zero to four.

(2) There exists only one critical velocity equal to the lowest phase velocity of waves in the Timoshenko beam if the loading frequency is zero, provided that the lowest phase velocity of waves is less than the shear wave velocity. There is no critical velocity for the loading frequency which is equal to zero if the lowest phase velocity of waves is equal to the shear wave velocity.

(3) The shear wave velocity $v_{c 1}$ is always one critical velocity and the longitudinal wave velocity $v_{c 2}$ cannot be the critical velocity for any loading frequency if $v_{1} / v_{2}<1 / 2$ and $\omega_{c 1} / \omega_{c 2}<\sqrt{2} / 2$, where $\omega_{c 1}$ and $\omega_{c 2}$ are two cutoff frequencies in the Timoshenko beam. 


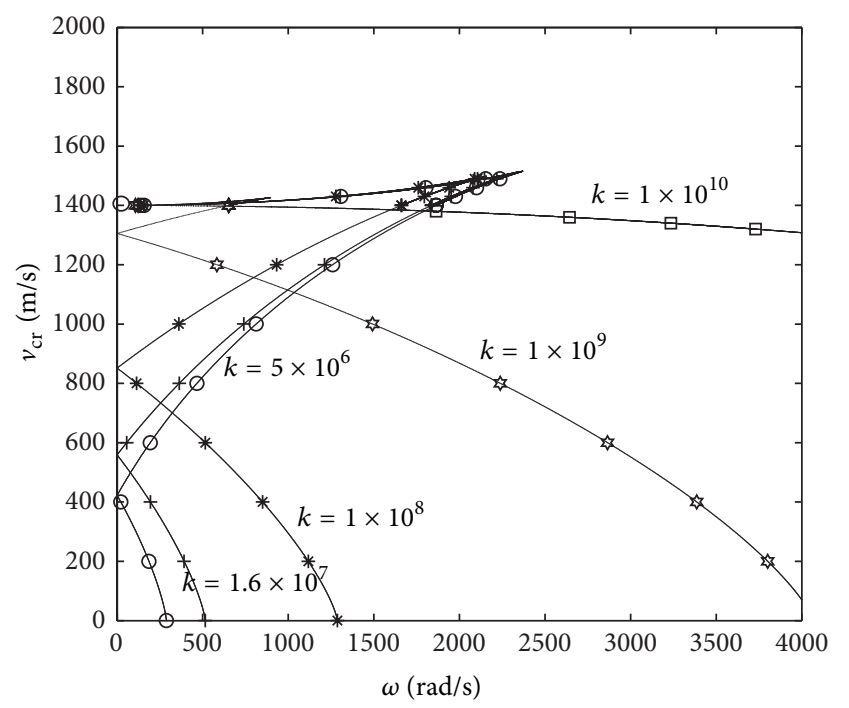

FIGURE 7: Critical velocity $v_{\text {cr }}$ versus loading frequency $\omega$ for different foundation stiffness $k\left(\mathrm{~N} / \mathrm{m}^{2}\right)$.

Numerical simulations illustrate that this result may still stand even when $\omega_{c 1} / \omega_{c 2}>\sqrt{2} / 2$. Moreover, all critical velocities for the Timoshenko beam are less than the longitudinal wave velocity $v_{c 2}$ no matter how large the foundation stiffness is or how high the loading frequency is.

(4) Numerical simulations for the European high-speed rail indicate that the maximum value of the group velocity $U_{1}$ in Timoshenko beam is just in the range of gouging of rails. Thus, $U_{1 \text { max }}$ (the maximum value of $U_{1}$ ) should be one "dangerous" velocity for the moving load in launching process, which has never been referred to in previous work.

\section{Appendix}

\section{Phase and Group Velocities of Waves in the Timoshenko Beam}

According to (11), the free vibration of the Timoshenko beam on the elastic foundation is described by

$$
\begin{gathered}
\frac{\partial^{4} \psi}{\partial t^{4}}+\frac{E \mu G A}{m \rho} \frac{\partial^{4} \psi}{\partial x^{4}}-\frac{k E}{m \rho} \frac{\partial^{2} \psi}{\partial x^{2}}+\left(\frac{k}{m}+\frac{\mu G A}{\rho I}\right) \frac{\partial^{2} \psi}{\partial t^{2}} \\
-\left(\frac{\mu G A}{m}+\frac{E}{\rho}\right) \frac{\partial^{4} \psi}{\partial x^{2} \partial t^{2}}+\frac{k \mu G A}{m \rho I} \psi=0 .
\end{gathered}
$$

The slope of the Timoshenko beam subjected to a moving load can be sought in the following form:

$$
\psi=C e^{i(N x-\omega t)}
$$

where $C$ is a constant representing slope amplitude, $N$ is the wave number, and $\omega$ is the circle frequency. Substituting (A.2) into (A.1) and canceling the term $C e^{i(N x-\omega t)}$ yield

$$
\begin{aligned}
\omega^{4}- & {\left[\left(v_{c 1}^{2}+v_{c 2}^{2}\right) N^{2}+\omega_{c 1}^{2}+\omega_{c 2}^{2}\right] \omega^{2} } \\
& +v_{c 1}^{2} v_{c 2}^{2} N^{4}+\omega_{c 1}^{2} v_{c 2}^{2} N^{2}+\omega_{c 1}^{2} \omega_{c 2}^{2}=0
\end{aligned}
$$

where $v_{c 1}=\sqrt{\mu G A / m}, v_{c 2}=\sqrt{E / \rho}, \omega_{c 1}=\sqrt{k / m}$, and $\omega_{c 2}=$ $\sqrt{\mu G A / \rho I}$. The two real roots of (A.3) are

$$
\omega_{1,2}=\sqrt{\frac{1}{2}\left(Q_{1} \mp \sqrt{Q_{2}}\right)},
$$

where $Q_{1}=\left(v_{c 1}^{2}+v_{c 2}^{2}\right) N^{2}+\omega_{c 1}^{2}+\omega_{c 2}^{2}$ and $Q_{2}=\left(v_{c 1}^{2}-v_{c 2}^{2}\right)^{2} N^{4}+$ $2\left[\left(\omega_{c 1}^{2}+\omega_{c 2}^{2}\right) v_{c 1}^{2}+\left(\omega_{c 2}^{2}-\omega_{c 1}^{2}\right) v_{c 2}^{2}\right] N^{2}+\left(\omega_{c 1}^{2}-\omega_{c 2}^{2}\right)^{2}$.

From (A.4), $\omega_{1} \rightarrow \omega_{c 1}, \omega_{2} \rightarrow \omega_{c 2}$, if $N \rightarrow 0 . \omega_{c 1}$ and $\omega_{c 2}$ are called the cutoff frequencies [14]. The phase velocities $v_{1,2}$ and group velocities $U_{1,2}$ in the Timoshenko beam can be expressed by

$$
v_{1,2}=\frac{\omega_{1,2}}{N}, \quad U_{1,2}=\frac{d U_{1,2}}{d N},
$$

respectively. It is easy to prove that $v_{1} \rightarrow v_{c 1}, v_{2} \rightarrow v_{c 2}$, if $N \rightarrow \infty . v_{c 1}$ and $v_{c 2}$ are called the shear and longitudinal wave velocities, respectively.

\section{Conflict of Interests}

The authors declare that there is no conflict of interests regarding the publication of this paper.

\section{Authors' Contribution}

All authors carried out the proofreading of the paper. All authors conceived of the study and participated in its design and coordination. All authors read and approved the final paper.

\section{Acknowledgments}

This work is supported by the National Natural Science Foundation of China (Grant nos. 11002103 and 11302119), the State Key Program of National Natural Science of China (Grant no. 11032009), and the National Natural Science Foundation of China (Grant no. 11272236).

\section{References}

[1] J. T. Kenney, "Steady state vibrations of beam on elastic foundation for moving load," Journal of Applied Mechanics, vol. 21, pp. 359-364, 1954.

[2] V. V. Krylov, A. R. Dawson, M. E. Heelis, and A. C. Collop, "Rail movement and ground waves caused by highspeed trains approaching track-soil critical velocities," Proceedings of the Institution of Mechanical Engineers F Journal of Rail and Rapid Transit, vol. 214, no. 2, pp. 107-116, 2000. 
[3] S. Timoshenko, "Method of analysis of statical and dynamical stresses in rail," in Proceedings of the 2nd International Congress of Applied Mechanics, pp. 1-12, Zurich, Switzerland, 1927.

[4] K. B. Lewis and N. V. Nechitailo, "Transient resonance in hypervelocity launchers at critical velocities," IEEE Transactions on Magnetics, vol. 43, no. 1, pp. 157-162, 2007.

[5] S. H. Crandall, "The Timoshenko beam on an elastic foundation," in Proceedings of the 3rd Midwestern Conference on Solid Mechanics, pp. 146-159, 1957.

[6] A. L. Florence, "Traveling force on a Timoshenko beam," ASME Journal of Applied Mechanics, vol. 32, no. 2, pp. 351-359, 1965.

[7] C. R. Steele, "The Timoshenko beam with a moving load," Journal of Applied Mechanics, vol. 35, pp. 481-488, 1968.

[8] S. Chonan, "Moving harmonic load on an elastically supported Timoshenko beam," Journal of Applied Mathematics and Mechanics, vol. 58, no. 1, pp. 9-15, 1978.

[9] Y.-H. Chen, Y.-H. Huang, and C.-T. Shih, "Response of an infinite tomoshenko beam on a viscoelastic foundation to a harmonic moving load," Journal of Sound and Vibration, vol. 241, no. 5, pp. 809-824, 2001.

[10] I. R. McNab, F. Stefani, M. Crawford et al., "Development of a naval railgun," IEEE Transactions on Magnetics, vol. 41, no. 1, pp. 206-210, 2005.

[11] L. Yang, X. Hou, and Z. Zeng, "A complete discrimination system for polynomials," Science in China E, vol. 39, no. 6, pp. 628-646, 1996.

[12] L. Yang, X. R. Hou, and Z. B. Zeng, "An alternative algorithm for determining the number of real roots of a polynomial," in Proceedings of the International Workshop on Logic and Software Engineering, World Scientific, Singapore, 1996.

[13] M. H. Kargarnovin and D. Younesian, "Dynamics of Timoshenko beams on Pasternak foundation under moving load," Mechanics Research Communications, vol. 31, no. 6, pp. 713-723, 2004.

[14] J. D. Achenbach, Wave Propagation in Elastic Solids, NorthHolland, New York, NY, USA, 1973.

[15] P. M. Fitzpatrick, Advanced Calculus, Thomson, Brooks/Cole, 2nd edition, 2006.

[16] A. V. Metrikine and H. A. Dieterman, "Lateral vibrations of an axially compressed beam on an elastic half-space due to a moving lateral load," European Journal of Mechanics A/Solids, vol. 18, no. 1, pp. 147-158, 1999. 

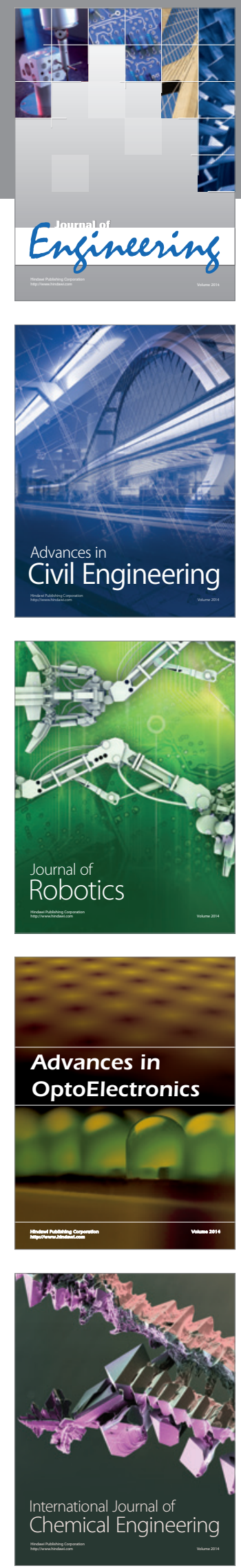

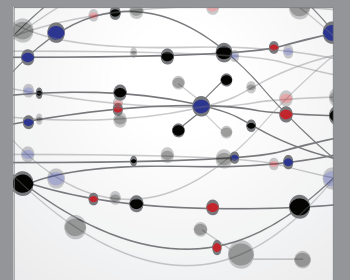

The Scientific World Journal
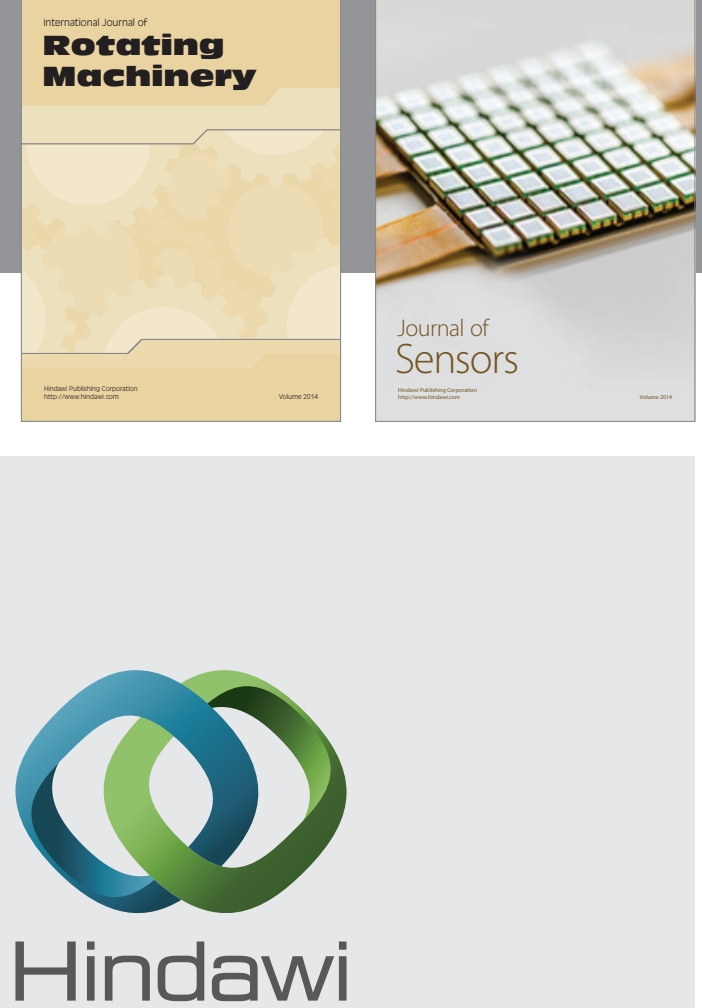

Submit your manuscripts at http://www.hindawi.com
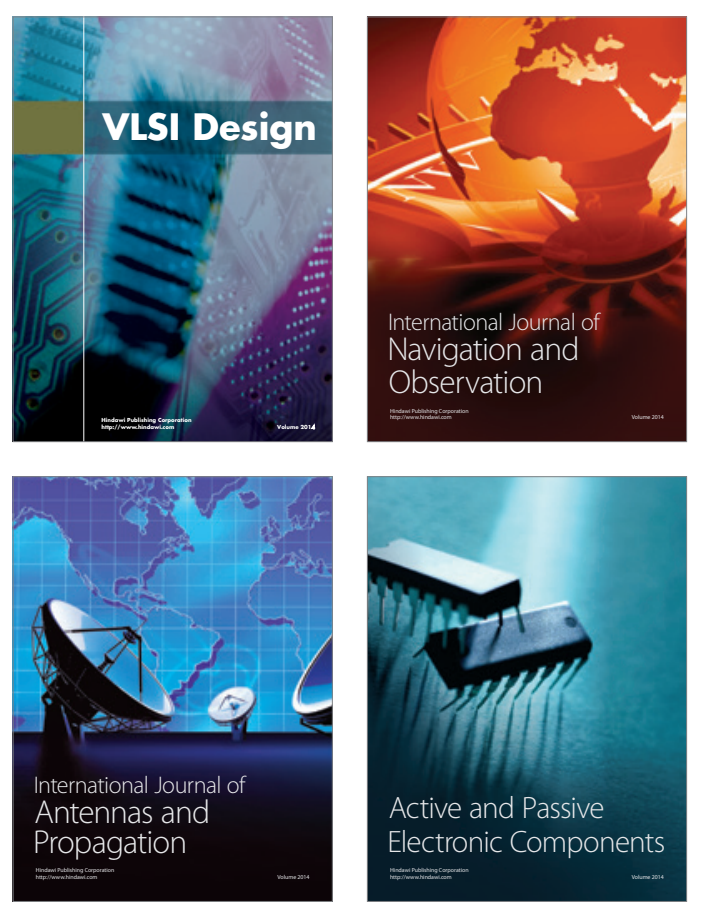
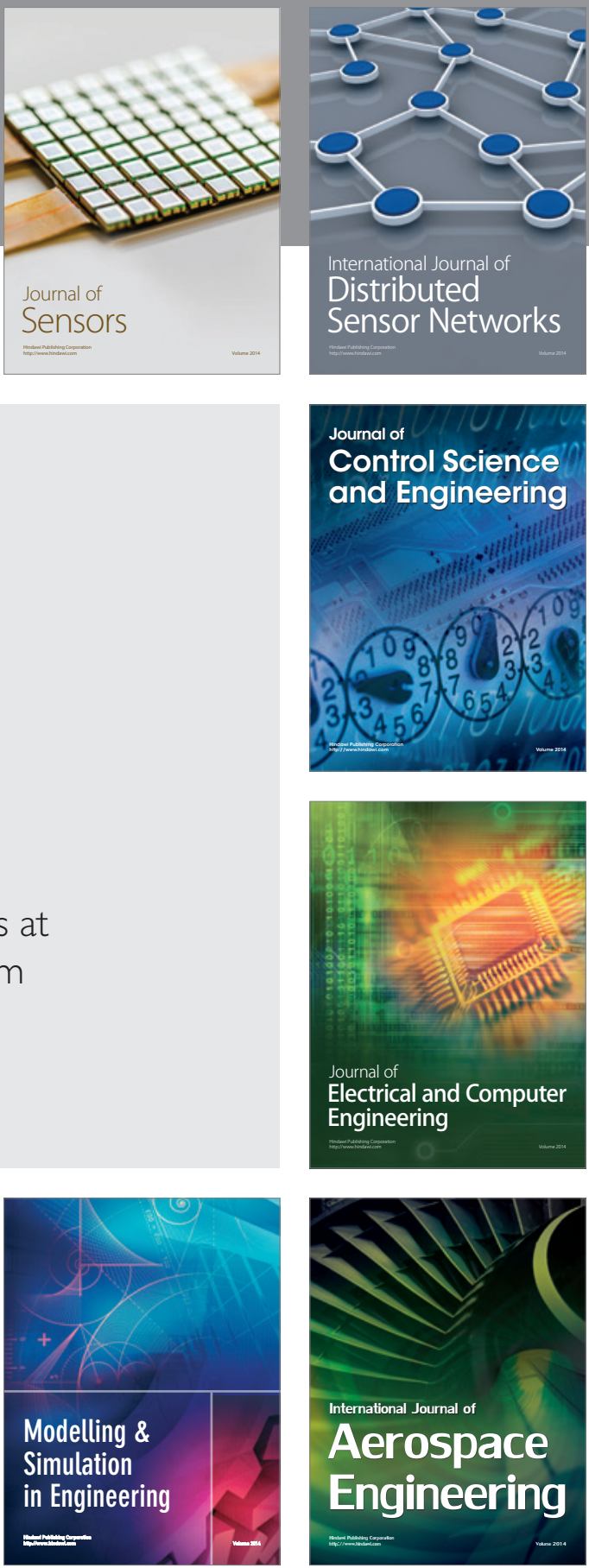

Journal of

Control Science

and Engineering
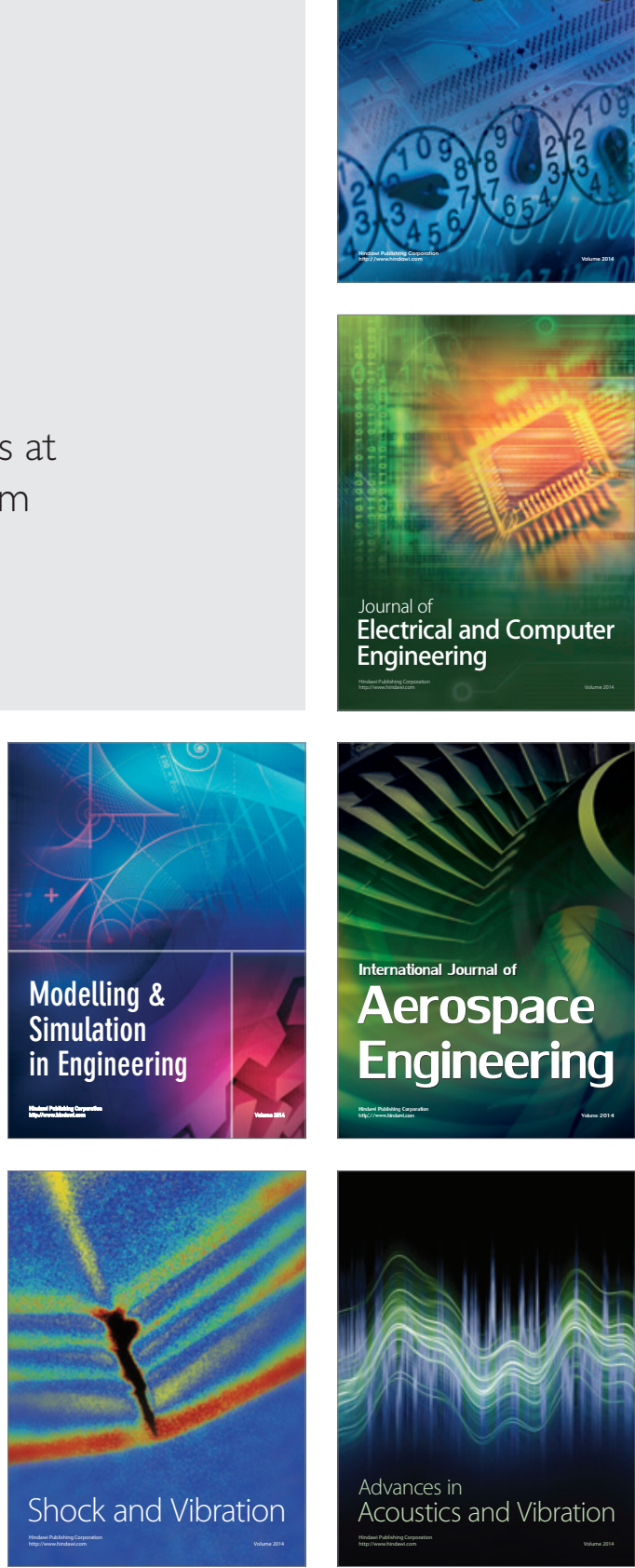